\title{
OPTIMIZED CLUSTER RECONSTRUCTION
}

\author{
STELLA SEITZ \\ Max-Planck-Institut für Astrophysik \\ Postfach 1523, D-85740 Garching, Germany
}

\begin{abstract}
We outline the noise-filtering finite-field inversion kernel and the regularized maximum likelihood methods for cluster reconstruction.
\end{abstract}

Unbiased finite-field mass reconstructions start from the relation $\nabla K=$ $\mathbf{u}$, where $K=\ln (1-\kappa)$, and the vector field $\mathbf{u}$ is a combination of the observable reduced shear $g=\gamma /(1-\kappa)$ and derivatives of it. Estimates of $K$ are thus obtained up to an arbitrary constant by averaging line integrals of observables. Noise degrades $\mathbf{u}$ to a combination of a gradient and a rotational field $\mathbf{u}=\nabla \tilde{K}+\mathbf{R}$, where only the rotational ('noise') component is treated differently by different line averaging methods. Requiring that the contribution from $\mathbf{R}$ is filtered out in the reconstruction is therefore a sensible requirement. For this, the above decomposition has to be specified uniquely by a boundary condition for $\mathbf{R}$ : for ideal data, where $\mathbf{u}$ is indeed a gradient field, $\mathbf{R}$ has to vanish identically; this implies that in this case the scalar field from which $\mathbf{R}$ can be derived has to be constant everywhere, in particular on the boundary. This boundary condition is consistent with the assumption that in the general case there should not be a systematic rotational component in the observed region $\mathcal{U}:\langle\mathbf{R}>\mathcal{U}=0$. Since $K$ and $\mathbf{u}$ are linearly related, we can make the ansatz that $K(\boldsymbol{\theta})-\bar{K}=$ $\int_{\mathcal{U}} \mathrm{d}^{2} \theta^{\prime} \mathbf{H}\left(\boldsymbol{\theta}^{\prime}, \boldsymbol{\theta}\right) \cdot \mathbf{u}\left(\boldsymbol{\theta}^{\prime}\right)$, where the additive constant $\bar{K}$ is chosen to be the average of $K$ over $\mathcal{U}$. We now replace $\mathbf{u}$ by its decomposition in gradient and rotational field and integrate by parts. Boundary terms vanish if we require that $\mathbf{H} \cdot \mathbf{n}=0$ on the boundary $\partial \mathcal{U}$, and the rotational component is 'filtered out' if $\mathbf{H}$ is a gradient field, $\mathbf{H}=\nabla \mathcal{L}$. With these requirements, the ansatz holds iff $\Delta \mathcal{L}\left(\boldsymbol{\theta}^{\prime}, \boldsymbol{\theta}\right)=\nabla \cdot \mathbf{H}\left(\boldsymbol{\theta}^{\prime}, \boldsymbol{\theta}\right)=-\delta\left(\boldsymbol{\theta}^{\prime}-\boldsymbol{\theta}\right)+\frac{1}{A}$, where $A$ is the area enclosed by $\partial \mathcal{U}$. Together with $\mathbf{H} \cdot \mathbf{n}=0$ this differential equation defines a Neumann boundary problem for $\mathcal{L}$, which implies an unique solution for $\mathbf{H}$. For a rectangular data field we have compared (see 
Seitz \& Schneider 1995 and references therein) reconstructions using the new kernel with earlier finite-field reconstructions of Schneider and Kaiser et al. or Bartelmann, hereafter named KSFWB/B, and the classical KSreconstruction. The ranking of the unbiased finite-field reconstructions in terms of the power spectrum of the error of the reconstructed $K$-field is always the following: the noise filtering reconstruction is the best, followed by the Schneider and KSFWB/B reconstructions. A clear peak of the power spectrum at large wavelengths reflects the artifacts introduced by the KSreconstruction.

I now outline the maximum likelihood reconstruction technique developed with Bartelmann, Schneider and Narayan: this method uses shape and magnification information (and possibly any other information) with the same weight simultaneously, it does not require the choice of a smoothing length and finally yields an objective measurement for the quality of the reconstructed mass profile. We define a model by choosing the deflection potential on a grid and calculate the mass density, shear and magnification, and finally the expectation value of the observables at every galaxy position and compare it to the 'measured' observables. The likelihood or, in the case of gaussian probability distributions (which we consider for simplicity), the $\chi^{2}$ per galaxy, measures the quality of the model considered. One now can minimize the $\chi^{2}$ to obtain a model which 'best' fits the data; this has a $\chi^{2}$ per galaxy considerably below unity, which illustrates that the data are 'overfitted'; the corresponding surface mass density shows strong short scale fluctuations, to fit the noise as closely as possible. To avoid this, we add to the $\chi^{2}$ a regularization term proportional to the sum of squares of derivatives of $\kappa$ at every grid point and minimize the sum. These derivatives can be of first or higher order, or combinations of these. The factor of proportionality must be adjusted such, that at the minimum, the $\chi^{2}$ for the shape and size and/or number-density distribution is equal to one. Any regularization contains a prejudice or prior knowledge about the mass distribution generating the observables. A regularization which is not compatible with the lens reveals itself immediately, since then the $\chi^{2}$ for the shape and magnification distribution can not become equal to one at the same time, or the $\chi^{2}$ locally becomes much larger then 1 (e.g. non-resolved substructure).

Acknowledgements: This work was supported by the "Sonderforschungsbereich 375-95 für Astro-Teilchenphysik" der Deutschen Forschungsgemeinschaft.

\section{References}

Seitz, S. \& Schneider, P. 1995, A\&A, in press. 